\title{
Séminaires / Seminari 2009
}

\section{Ouverture et reprise d'un cabinet médical}

Contenu (Détails $\rightarrow$ www.fmhservices.ch)

Business plan - Aménagement - Estimation d'un cabinet - Administration d'un cabinet médical Assurances - Passage du statut de salarié à celui d'indépendant et fiscalité.

\section{Sponsors}

Les coûts sont pris en charge par divers sponsors (voir www.fmhservices.ch).

\section{Dates}

$\begin{array}{lll}\text { K20 } & \begin{array}{l}\text { Jeudi } 5 \text { mars } 2009 \\ \text { Lausanne } 17.00-21.30 ~ h\end{array} & \begin{array}{l}\text { World Trade } \\ \text { Center }\end{array} \\ \text { K21 } & \begin{array}{l}\text { Jeudi } 18 \text { juin } 2009 \\ \text { Neuchâtel } 17.00-21.30 ~ h\end{array} & \text { La Maladière } \\ \text { K22 } & \begin{array}{l}\text { Jeudi } 3 \text { septembre } 2009 \\ \text { Genève } 17.00-21.30 ~ h\end{array} & \begin{array}{l}\text { Ramada } \\ \text { Park Hôtel }\end{array} \\ \text { K23 } & \begin{array}{l}\text { Jeudi } 19 \text { novembre 2009 } \\ \text { Fribourg } 17.00-21.30 ~ h\end{array} & \begin{array}{l}\text { Au Parc } \\ \text { Hôtel }\end{array}\end{array}$

\section{Remise d'un cabinet médical}

\section{Contenu (Détails $\rightarrow$ www.fmhservices.ch)}

Aspects juridiques - Estimation d'inventaire et goodwill d'un cabinet - Assurances - Conséquences fiscales d'une remise.

\section{Sponsors}

Les coûts sont pris en charge par divers sponsors (voir www.fmhservices.ch).

\section{Dates}

$\begin{array}{lll}\text { K24 } & \begin{array}{l}\text { Jeudi } 23 \text { avril } 2009 \\ \text { Lausanne } 17.00-21.30 \mathrm{~h}\end{array} & \begin{array}{l}\text { World Trade } \\ \text { Center }\end{array} \\ \text { K25 } & \begin{array}{l}\text { Jeudi } 12 \text { novembre } 2009 \\ \text { Genève } \quad 17.00-21.30 ~ h\end{array} & \begin{array}{l}\text { Ramada } \\ \text { Park Hôtel }\end{array}\end{array}$

\section{0 ans avant le départ à la retraite}

\section{Remarque}

Les adresses des participants aux séminaires dont les coûts sont couverts en partie ou totalement par des sponsors sont communiquées aux sponsors concernés.

\section{Conditions d'annulation}

Un montant est perçu pour une absence ou une annulation. Il est de:

- Fr. 50.- par personne dans les 15 jours avant;

- Fr. 100.- par personne dans les

7 jours avant le début du séminaire.

\section{Contenu}

Ma rente va-t-elle suffire? Questions relatives $\mathrm{au}$ besoin éventuel de capital, aux solutions possibles de prévoyance professionnelle et à une démarche fiscale optimalisée. Fiduciaire et pré- voyance vieillesse? Possibilités d'harmoniser une planification idéale du départ à la retraite avec la vente éventuelle du cabinet médical afin de réaliser des économies fiscales importantes. Planification de la prévoyance vieillesse! Estimation précise des besoins futurs sur la base des possibilités de planification existantes et planning optimal des futurs revenus tenant compte du montant, des impôts et de la sécurité.

\section{Coûts}

Fr. 100.- (y compris documents de cours et collations).

\section{Dates}

\begin{tabular}{|c|c|c|}
\hline \multirow[t]{2}{*}{ K40 } & Jeudi 25 juin 2009 & \multirow{2}{*}{$\begin{array}{l}\text { Ramada } \\
\text { Park Hôtel }\end{array}$} \\
\hline & $18.00-20.30 \mathrm{~h}$ & \\
\hline \multirow[t]{2}{*}{ K41 } & Jeudi 27 août 2009 & World Trade \\
\hline & $18.00-20.30 \mathrm{~h}$ & Center \\
\hline
\end{tabular}

\section{Contabilità e fiscalità (Ticino)}

\section{Contenuto}

L'obiettivo di questo seminario è di dare al medico le conoscenze necessarie per utilizzare autonomamente lo strumento contabile e per operare con più efficienza ed economicità, nonché far comprendere al medico il significato della pianificazione fiscale.

Mediante esempi pratici elaborati in gruppo, sarà illustrato in maniera semplice, ma completa il sistema di gestione della contabilità di uno studio medico con effetto anche sulla pianificazione fiscale.

\section{Spese}

Gratuito per membri FMH.

\section{Date}

$\begin{array}{lll}\text { K50 } & \begin{array}{l}\text { Giovedi } 7 \text { maggio } 2009 \\ \text { Chiasso dalle } 9.00 \text { alle 16.00 }\end{array} & \begin{array}{l}\text { FMH } \\ \text { Fiduciaria } \\ \text { Services }\end{array} \\ \text { K51 } & \begin{array}{l}\text { Giovedi } 8 \text { ottobre } 2009 \\ \text { Chiasso dalle } 9.00 \text { alle } 16.00\end{array} & \begin{array}{l}\text { FMH } \\ \text { Fiduciaria } \\ \text { Services }\end{array}\end{array}$

\section{Inscription et information}

Internet www.fmhservices.ch ou FMH Consulting Services, Sandra Stadelmann, Burghöhe 1, 6208 Oberkirch, Tél. 04192500 77, Fax 041 9210586. 